\title{
Gamma Ray Bursts as Death of Magnetized Neutron Stars
}

\author{
Hitoshi HANAMI \\ Physics Section, Faculty of Humanities and Social Sciences, Iwate \\ University, Morioka 020 JAPAN
}

\begin{abstract}
We propose magnetic cannon ball mechanism in which the collapse of a magnetosphere onto a black hole can generate strong outward Poynting flux which can drive a baryon-free fireball. This process can occur at the final collapsing phase of a neutron star with strong magnetic field. The magnetic cannon ball can drive a relativistic outflow without the rotation of the central object. This baryon-free process can explain gamma-ray bursts as the final phase of dead pulsars.
\end{abstract}

\section{Gamma Ray Bursts and Magnetized Neutron Stars}

The observations by BATSE presented that the distribution of classical gamma ray bursts (CGRBs) is isotropical on the sky, but inhomogeneous (Meegan et al. 1992), which implies that the origin are cosmological or in the extended halo of the Galaxy. The behavior of CGRBs are similar to that of active galactic nuclei (AGNs); the rapid variability $\left(<10^{-3} \mathrm{~s}\right)$, power-law spectrum features without a cutoff due to self-absorption via the pair production $\gamma+\gamma \rightarrow e^{+}+e^{-}$. Then, it is believed that fireball process with relativistic expansion can play important rules in the physical process of CGRBs as the superluminal motion in AGNs.

There are some scenarios for releasing the energy into a fireball with the gravitational collapse to a NS or a $\mathrm{BH}$. In most of these scenarios, two different processes were proposed for driving a fireball; neutrino pairs driven due to $\nu+$ $\bar{\nu} \rightarrow 2 \gamma \rightarrow e^{+}+e^{-}$, and magnetic driven due to the Poynting flux from a rapid rotator like a millisecond pulsar or a rotating disc around a $\mathrm{BH}$ with unusually strong magnetic fields $B \simeq 10^{15} \mathrm{G}$.

The baryon fraction, however, should be even smaller than $10^{-6} M_{\odot}$ for required relativistic boost $(\gamma>100)$ ( Mészáros \& Rees 1993). Furthermore, these fireball models cannot explain observed cyclotron absorption lines (Murakami et al. 1988; Fenimore et al. 1988; Yoshida et al. 1991), which are consistent with the existence of magnetic field $\simeq 10^{12} \mathrm{G}$. Magnetic driven models may have some advantages to explain the cyclotron features.

A strong magnetic rotator requires millisecond period for the cosmological origin. We worry about that the strong magnetic field may trade off the millisecond rotation. In the magnetic rapid rotators, the magnetic energy itself $\simeq 10^{49}\left(B / 10^{16} \mathrm{G}\right)\left(R / 10^{6} \mathrm{~cm}\right)^{3}$ erg is nearly comparable to their rotational energy and the required energy for the cosmological origin. The magnetic energy $\simeq 10^{44}\left(B / 10^{13} \mathrm{G}\right)^{2} \mathrm{erg}$ of ordinary pulsars is efficient for a CGRB in the Galactic 
halo. Then, we will consider a mechanism in which the magnetic energy itself is converted into $\gamma$-rays.

\section{Magnetic Cannon Ball}

When a NS with the magnetosphere collapses into a $\mathrm{BH}$, the current generating the magnetic fields also falls into the horizon of the $\mathrm{BH}$, so that the magnetosphere decaying induces outward Poynting flux in the our result (Hanami 1996). The most of magnetic energy is carried by the electromagnetic wave radiation. Like pulsars, the sequential processes produce relativistic $e^{+}+e^{-}$pair plasma. The emitting wave interacts with the created $e^{+}+e^{-}$pair plasma. The wave energy can be converted to that of turbulent pair plasma even if the wave frequency is lower than the plasma frequency $\omega_{p}=\left(4 \pi n e^{2} / m\right)^{1 / 2} \simeq 9000 n^{1 / 2} \mathrm{~s}^{-1}$. This picture is similar to thermal pair plasma fireball due to neutrino deposition. We will call the our presented model as magnetic cannon ball.

\section{Conclusions and Discussion}

The released energy due to the magnetic cannon ball is given as $E=1.4 \times$ $10^{48} \mathrm{erg}\left(B_{\text {init }} / 3 \times 10^{15} \mathrm{G}\right)^{2}\left(r_{\text {init }} / 10^{6} \mathrm{~cm}\right)^{-2}\left(r_{\text {final }} / 10^{5} \mathrm{~cm}\right)^{-1}$. This value is less than the required energy $\simeq 10^{51}$ erg for a spherically symmetric fireball at cosmological distance. As discussed in models of AGN and minijet model for CGRBs, however, relativistic beaming with the Lorentz factor $\Gamma$ can exclude this problem since the required energy can decrease as small beam opening angle $\theta \simeq 1 / \Gamma$. After pair plasma production, a magnetic cannon ball can be expanding with the magnetic field according to magnetohydrodynamics. The magnetic field frozen in the cannon ball decreases as $B(R) \simeq B_{0}\left(R / R_{0}\right)^{-2}$, where $R$ is the radius of the cannon ball and ' 0 ' means the initial value. Following to the dynamics of fireballs, we can also consider the expansion of the magnetic cannon ball as $\left(R / R_{0}\right) \simeq \Gamma$ (e.g. Mészáros \& Rees 1993). If the photons of a burst is released at the expanding phase, cyclotron absorption occurs due to the remnant magnetic field $B \simeq 3 \times 10^{11} \mathrm{G}\left(B_{0} / 3 \times 10^{15} \mathrm{G}\right)(\Gamma / 100)^{-2}$. This is convenient for explaining the observed cyclotron absorptions (Murakami et al. 1988; Fenimore et al. 1988; Yoshida et al. 1991). The magnetic field in the cannon ball can confine the high energy protons $(\gamma \simeq 300)$ which are required for delayed photons $(>25 \mathrm{GeV}$ ) following a burst on 1994 February 17. The final remnant in the model should be a black hole. It implies that any gamma-ray bursts can have no optical counter part if they do not have a companion in the binary.

\section{References}

Hanami,H'. 1996, ApJ, submitted

Meegan, C.A. et al. 1992, Nature, 355, 143

Mészáros,P. \& Rees, M. 1993, ApJ, 405, 278

Murakami, T. et al. 1994, Nature, 368, 127 